\title{
Near Field Communication Tag-based System in Retail Marketing
}

\author{
Qiyan Wang, Sabah Mohammed and Jinan Fiaidhi \\ Department of Computer Science, Lakehead University \\ 955 Oliver Road, Thunder Bay, Ontario, Canada \\ \{qwang4, sabah.mohammed, jfiaidhi\}@lakeheadu.ca
}

\begin{abstract}
Near Field Communication (NFC) is a short-range wireless communication technology capable of two way communication. As an emerging technology, it has been widely used for payment (a debit and credit) for example, or even in medicine ( $a$ tag in your skin could send vital signs to your smartphone). In this paper, we describe a mobile prototype system using NFC for retail marketing. The system is responsible for collecting data about customers using NFC tags to analyze their personal purchase behavior. The direct outcome is to increase revenue of retailors from customers' repeated purchases by pushing customized marketing activities like discount vouchers and deal information based on the outcome of the analysis. We fully describe the technical including architectures and basic workflow, and explain the details of use cases.
\end{abstract}

Keywords: Near Field Communication; NFC tag; Data collection; Data analysis; Retail marketing

\section{Introduction}

NFC is a short distance wireless communication technology standards. It can create a wireless network mobile device quickly and automatically so that the electronic device can communicate in short range. Short distance interaction of NFC greatly simplifies the authentication and identification process. Besides, it makes the electronic equipment communicate with each other more direct, safely and clearly. The technology behind NFC allows a device, known as a reader, interrogator, or active device, to create a radio frequency current that communicates with another NFC compatible device or a small NFC tag holding the information the reader wants. Passive devices, such as the NFC tag in smart posters, store information and communicate with the reader but do not actively read other devices. Peer-to-peer communication through two active devices is also a possibility with NFC. This allows both devices to send and receive information [1].

NFC tags communicate using the ISO 14443 type A and B wireless standards, which is the international standard for contact-less smartcards, used on many public transportation systems. This is why NFC devices can be used with existing contact-less technologies, such as card payment points. There are a range of different tag types available, each offering different storage levels and transfer speeds. The strongest argument in favor of NFC, over other forms of short range wireless communication, is that tags are incredibly cheap to make and maintain, but can still be used for a wide range of applications. With very simply circuitry and very few components, NFC tags can be produced on mass for very low unit costs. Combine low costs with the absence of any power requirements, and you have a cheap yet effective way of quickly communicating with other smart devices. From launching applications, to exchanging web addresses and purchasing a rail ticket, NFC aims to make our lives that little bit more convenient just by using our smartphones [2].

In this paper, we propose a NFC Tag-based System targeting the NFC tag read records from customers and analyzes individual shopping behavior and preference. From 
merchants point, the main advantage of the system is that they are able to customize business activities which are more attractive to every customer so that increase revenue in an efficient way. In the following section, we introduce the characteristics of our application and compare it with some NFC-based implementations. Section 3 presents the overview of the system, describing the architecture, main components, and workflow. In Section 4, we explain two real-world use cases to show the advantages and potential marketing value of the system. Finally, the conclusion of this paper can be found in Section 6.

\section{Related Researches}

Near-field communication (NFC) is a set of communication protocols that enable two electronic devices, one of which is usually a portable device such as a smartphone, to establish communication by bringing them within $4 \mathrm{~cm}$ (1.6 in) of each other. NFC devices are used in contactless payment systems, similar to those used in credit cards and electronic ticket smartcards and allow mobile payment to replace/supplement these systems. NFC is used for social networking, medical Alert systems such as Tap2Tag ${ }^{1}$, for sharing contacts, photos, videos or files. NFC-enabled devices can act as electronic identity documents and keycards. NFC offers a low-speed connection with simple setup that can be used to bootstrap more capable wireless connections ${ }^{2}$. NFC-equipped smart phones usually come with a wallet software that can store coupons, loyalty points, and payment account information. Consumers carry and manage their coupons and promotions in the mobile wallet. They can access personalized coupons and promotions by searching through the wallet or by tapping the NFC phone on a smart poster or shelf tag. With a consumer's permission, retailers can also send coupons or promotions "over the air" directly to the phone. Coupons are redeemed electronically, by tapping the phone on a contactless-enabled POS reader. However employing this new technology retail marketing requires more considerations. In the following subsections we describe the related research regarding some of the relevant work.

\subsection{Loyalty Application Challenges}

For most retailors, growth means increase in revenue, which is mainly caused by saving current customers and acquiring new customers as well as retaining old customers. Thus, analyzing the customer purchase behaviors is critical to predict expected behaviors of individual customer and using the result to perform efficient marketing activities. With the popularity of smart mobile devices, we can find some applications targeting specific retailer's loyalty program. Due to the existing of these applications, not only customers become much more convenient to collect loyalty points and save money, but also it promotes retailers' revenue by customers' repeat purchases. However, some problems are still existing.

- So many loyalty programs: Even though loyalty applications installed in smartphone has replaced plastic cards. However, there are still too many similar programs with different point collection rules, login information and application designs, which always make customer confused.

- Tedious payment process: When customer pays at cashier counter, he or she has to open the loyalty application, and then present credit or debit card. Although the process has been simplified comparing decades ago, it is not efficient enough in recent fast-pace society.

\footnotetext{
${ }^{1}$ https://www.tap2tag.me/about

${ }^{2}$ https://en.wikipedia.org/wiki/Near_field_communication
} 
- Security: With smartphone becoming more central to daily life and work, it is suffering much more risk than before. However, people have not much knowledge about how to protect their phones.

\subsection{Some NFC-based Solutions}

Based on the characteristics of Near Field Communication, there are many existing solutions, which basically can be divided into two categories: Card emulation application and NFC-based smart card system.

NFC allows mobile phones to emulate cards (i.e. store cards data and work as conventional loyalty cards), to read cards or tags and to communicate via Peer-to-Peer. These capabilities and the fact that mobile phones provide an interface for managing the data which was received and sent by them, are the main reasons why NFC is the technology chosen for developing a solution [3]. "Loyalty Systems over Near Field Communication (NFC)" from Diogo Simões, Technical University of Lisbon. He presents an integral and detailed solution for managing loyalty accounts through NFC technology. The concept of the solution is motivated by the fact that even though the current loyalty applications replace the traditional plastic cards, customers always only want to present the loyalty account when they at cashier and do not want to spend pay much attention to the functionalities in the applications. The main objective is to allow users to keep their accounts in a simple and safe way, supported by Near Field Communication enabled mobile phones. This solution states that if a user has access to any of his loyalty accounts he may need and he also can consult the cards information.

There is another paper - "NFC Loyal: A Beneficial Model to Promote Loyalty on Smart Cards of Mobile Devices", which introduces another NFC technology implementation. NFC adopted smart card as secure element (SE) to provide a secure area for the execution of multiple applications as well as storing sensitive data. In this study, the design of new way of NFC enabled loyalty system on smart cards of NFC mobiles. With this model, loyalty and payment applications share and exchange valuable information to obtain mutual financial outcomes [4]. By combining loyalty and payment applications, the payment process is finished by using one application, which is a huge simplification. At the same time, the security structure of NFC adopted smart card also protects customer from risk.

\subsection{NFC-based Solutions Weakness}

From the two papers, they all come up with solutions solving some problems in retail market using NFC technology. However, as the most critical role in retail marketing, data integration is always overlooked by mobile applications. Many customer loyalty programs focus on rewarding customers for spending a specific amount at a store, and do not collect detailed information about the customer and his purchases. By limiting the information you collect about your program participants, you're losing valuable opportunities to analyze trends and insights in your marketing and sales efforts, as well as the opportunity to market directly to customers through email, text, and mail correspondence [5]. In this study, we develop a NFC Tag-based System targeting customer's purchase and behavior data collection and analysis, which contributes to retail marketing decision and customized business activities. 


\section{NFC Tag-based System}

Many participants in the direct marketing and couponing industries may be affected by or play an active role in mobile marketing, including ${ }^{3}$ :

- Direct marketing agencies

- Advertising networks and exchanges

- Telemarketing companies

- Coupon printers, processors, and clearinghouses

- List compilers and sellers

- Analytical support providers

However, they are lacking a uniform platform for analyzing the collected tags and benefit from the consumer collected information. This section illustrates the required architecture for such framework.

\subsection{NFC Tag Usage Lifecycle}

In this system, NFC tag usage is defined at four statuses including Write, Read, Monitor and Analysis depending on the operation to the content in tag by different user. As a fully functional NFC Tag-based management system, it should be able to involve whole NFC tag usage lifecycle. Figure 1 shows a sequence diagram of the NFC tag usage lifecycle and operated by different user.

First of all, retailer uses the mobile application to write certain information, like product detail, discount activity or store location, into NFC tags and place them in-store or at some public areas with huge passenger traffic.

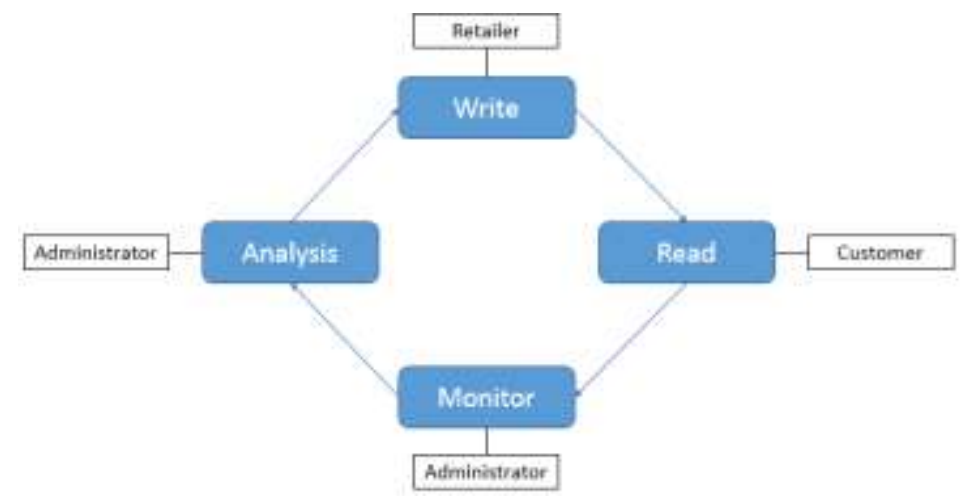

Figure 1. NFC Tag Usage Lifecycle

When customers want to know the detail of in-store activity, or find the store, they can simply approach the mobile device to the tag, its content shows on the device and starts corresponding build-in applications perform the content. For example, the store address in the NFC tag triggers navigator to locate the store automatically and lead customer to arrive at the destination after the device reads the tag. Every NFC tag usage from the mobile client is recorded and sent to database including the tag serial number, content saved into the tag, user name who execute the operation and the operation time. As an administrator, he or she monitors every usage record on the background server, which communicates with database.

From the NFC tag usage records, retailer summaries every customer's shopping preference and deeply excavate personal shopping behavior which is helpful to

3

https://www.securetechalliance.org/resources/pdf/Chip-Enabled_Mobile_Marketing_WP_092710. pdf 
customized personal retail activity. At the same time, the administrator can also choose specific tags and generate statistics about tags read time compare. At the last step of the lifecycle, retailer uses the mobile client update the content in the tag when it is out of date and starts a new data collection.

\subsection{Architecture and Implementation}

NFC Tag-based System is a management system based on Android platform concerning NFC tag and involving whole lifecycle of NFC tag usage. It is consisted of hardware (including NFC tags, server, mobile devices) and software (mobile client, database, management system).

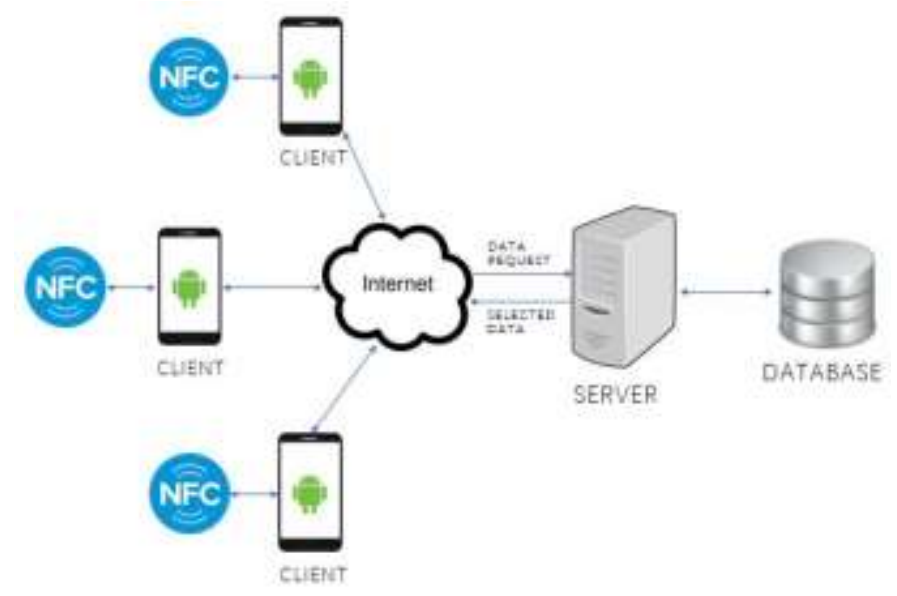

Figure 2. NFC Tag-based System Architecture

As shown in Figure 2, there are totally four parts in the whole platform: NFC tags, Android devices, server and database. NFC tag does not have its own power source. When device with the mobile client installed reads it, the passive tag absorbs a small portion of the energy emitted by the reader and starts sending modulated information when sufficient energy is acquired from the RF field generated by the device. At the same time, the reader (phone or tablet) will generate the corresponding usage record. Parse the NFC tag is simple:

- Figure out the MIME type or a URI that identifies the data payload in the tag

- Encapsulate the MIME type or URI and the payload into an intent

- Start an activity based on the intent

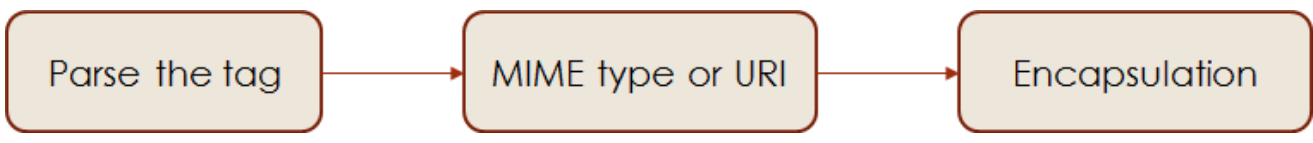

For each Android device, it needs to install the mobile client to communicate with NFC tags. To distinguish the source of each tag usage, the user login functionality is indispensable and triggers when the application is opened. after providing username and password, and checking with database, the main screens of mobile client are shown in Figure 3. Reading NFC tag is the most basic functionality and opened to every user. After approaching the tag to the device, the content shows on the Read tab (Figure 3a), based on the content category, the client triggers corresponding build-in application. Besides, the user information and password can be reviewed and edited on the Other tab (Figure 3c). As a retailer and administrator, it has permission to update the tag content by clicking category option (Figure 3b), typing the new one and pushing the Write button. For 
administrator, there are some more tag management option in Other tab like erase, lock or set password for NFC tag.

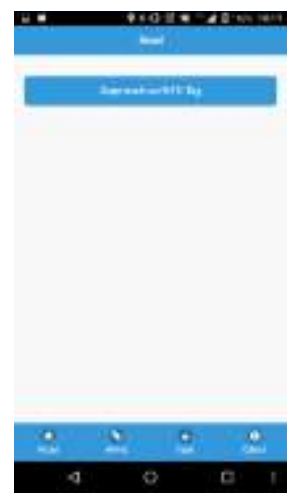

(a)

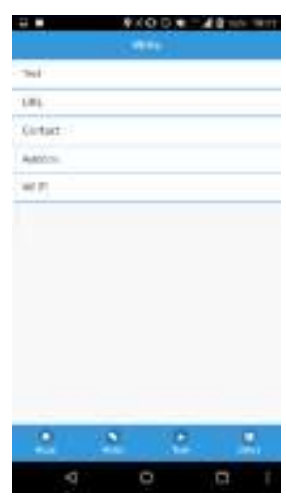

(b)

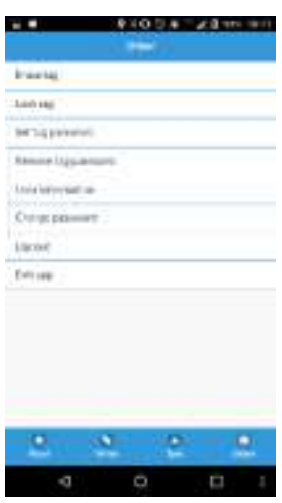

(c)

Figure 3. Snapchats of mobile client

When the tag dispatch system is done creating an intent that encapsulates the NFC tag and its identifying information, it sends the intent to an interested application that filters for the intent. If more than one application can handle the intent, the Activity Chooser is presented so the user can select the Activity (see the following figure for more details).

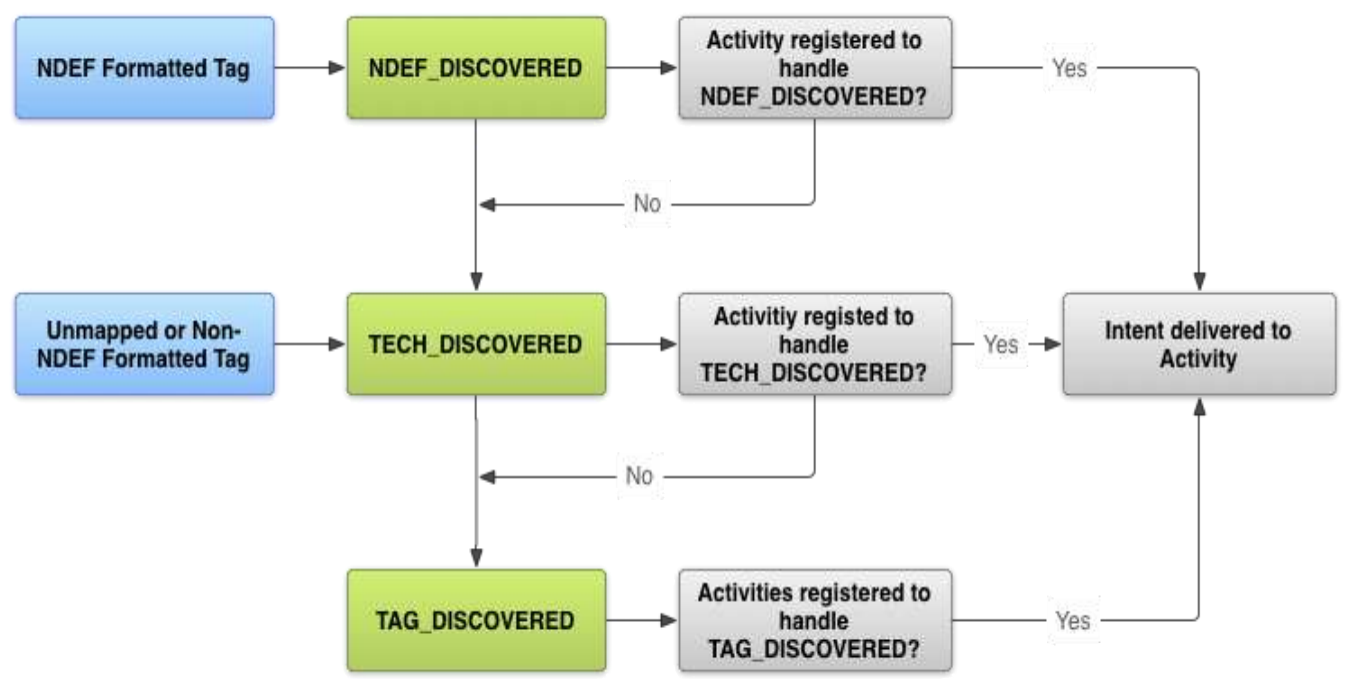

Mobile client keeps fetching the tag usage record and sends to database through server. In the database, each record is stored in the tag usage record table with certain organization. Server provides computers with services related to accessing and retrieving data from database. On the system management webpage, the data from database is retrieved and shown with designed format by running certain query. After administrator login, he can not only view the tag usage records but also execute some command to manage data like Add, Edit and Delete. After the data retrieved from database, the background of the server side is programmable to classify and count the desired data in by developer. And then, the processed statistics are presented on the server webpage. The mobile client components can be described as follows: 


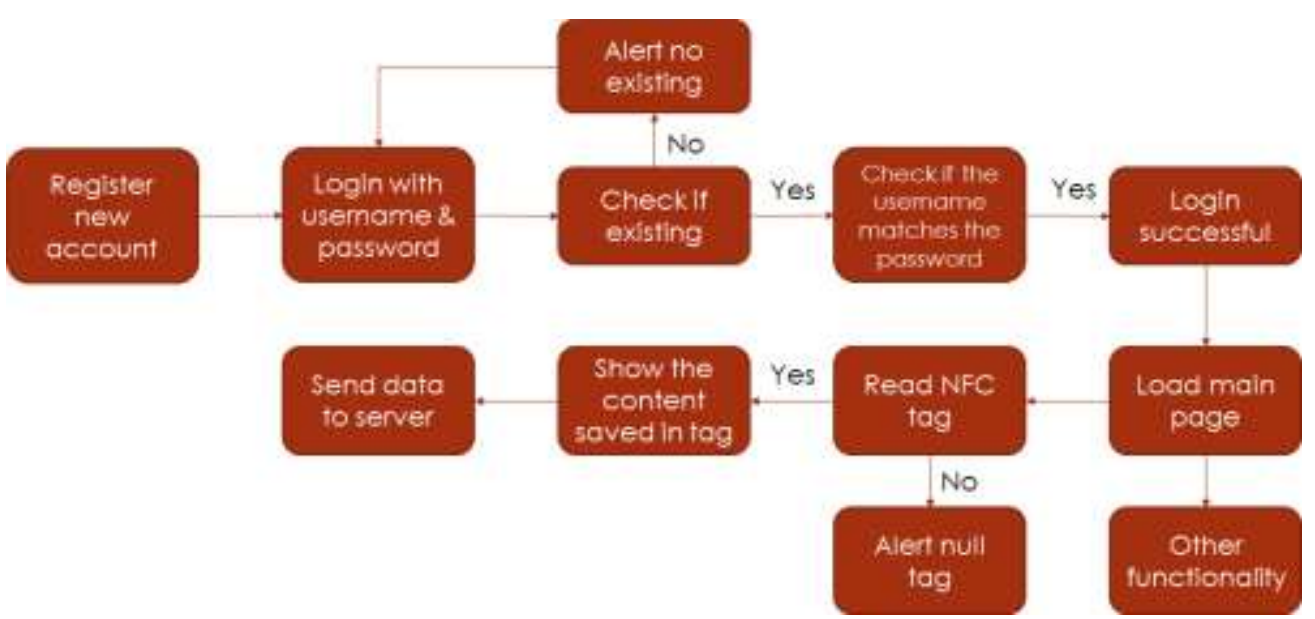

In the next section, we introduce the workflow and advantages of the system based on a real-world use case.

\section{System Application and Case Study}

The NFC Tag-based System acts as an end-to-end source of information, which provides shopping information to assist customer in choosing product, deal or finding store location. Customers only need to follow the scenarios:

- Download and install NFC Tag-based Application on the personal Andriod mobile device;

- Create new account or login the existing account;

- Choose a NFC tag with the interested information;

- Start the application and approach device to the tag;

- The content in the tag shows on the screen and triggers corresponding build-in application performing the content (Such as location information starts map application and navigates to the store, URL starts browser to open the webpage).

At the same time, the mobile application is also a customer's data collector and sends to database. In the background, data is used to compare retailers and analyze each customer's shopping behavior so that adjust marketing strategy and customize personal deal to meet every customer's need. Although the possible application occasions are multiple, in the following two cases, we apply the system to shopping mall and retail store by providing a range of related shopping information stored in multiple NFC tags and placing them into multi-locations to let as many as customers read the information using their mobile devices.

In the following sub-sections, we describe in detail the two case studies workflows and show the results of data analysis.

\subsection{Shopping Mall Retailer Directory}

When customers step into a shopping mall, most of them have their personal preferred retailers which will be the first to visit. Even though every shopping mall has the directory board to assist customers find them and know about the in-store activities, it is still not efficient and accurate enough. 


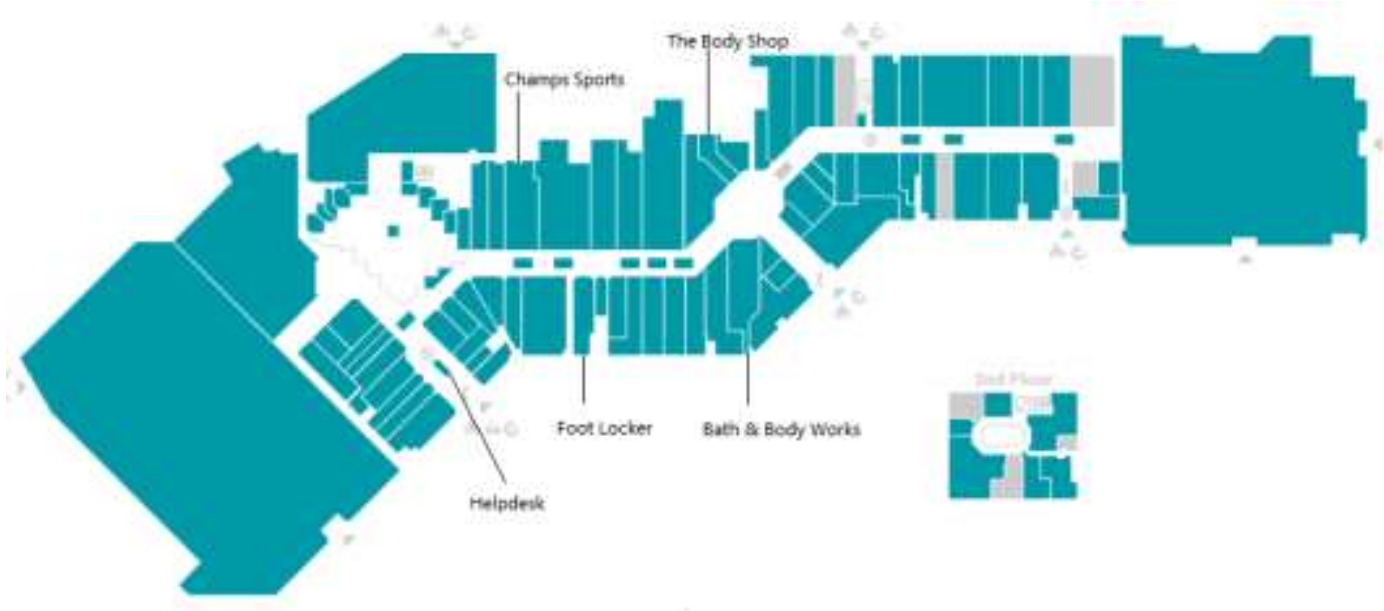

Figure 4. Map of Intercity Mall

In this case, we choose Intercity Shopping center in Thunder Bay and place multiple NFC tags saving location and deal information of Foot Locker, Champs Sports, The Body Shop and Bath \& Body Works at helpdesk (Figure 4). Customers are free to use their mobile devices to get the desired information following the scenarios we present above. After one hour, we login to the system management server and get the follow statistics.

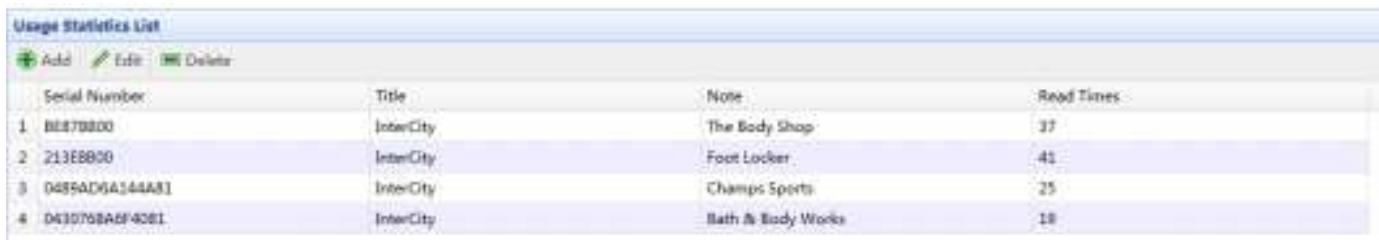

\section{Figure 5. Usage Statistics List}

In database, we utilize the unique serial number for each single NFC tag into a part of the tag read record. The NFC tags with the information, which we want to calculate their read times, are chose and show on the Usage Statistics List in the background server. After customer reads these tags, the server counts read time of each tag in the usage statistics list (Figure 5). In this case, based on the list, we draw charts for read times of each store. To make the compare straightforward, the stores are divided into two groups based on their product categories. In the first group (Figure 6a), The Body Shop and Bath $\&$ Body Works are all selling beauty related products with similar market positioning, we can conclude that customers prefer visiting The Body Shop rather than Bath \& Body Works. Similarly, Foot Lockers is more popular than Champs Sports although they all have sport related products (Figure 6b). From the results, the retailer manager can change the business strategy in time based on the clear compare, such as bigger discount activity or more product varieties. After a period, the new statistics will prove the effectivity of the applied changes. 


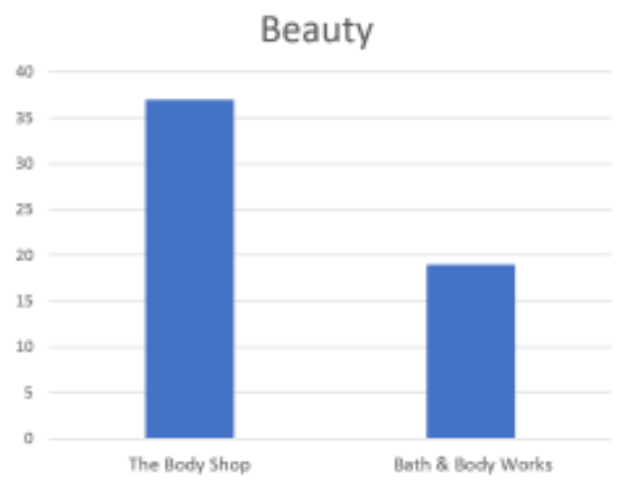

(a)

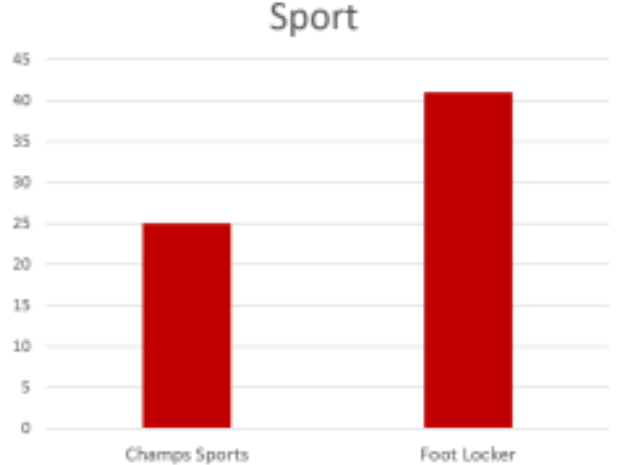

(b)

Figure 6. Charts for Four Retailers Compare

\subsection{In-store Deal Information}

Besides the stores compare in the previous case, each retailer also want to get more information about its customers. In this case, it is focusing on customers who enter the store and collect related data to analyze their shopping preferences. We place multiple NFC tags at different areas in Sears in Intercity Mall, and we write corresponding product information or in-store activities based on the area's products. When customer is interested in the details, he or she can use the personal device read the tag or get the deal at cashier. After one hour, we check the Tag Usage List and try to conclude the shopping preference for every customer in the records.

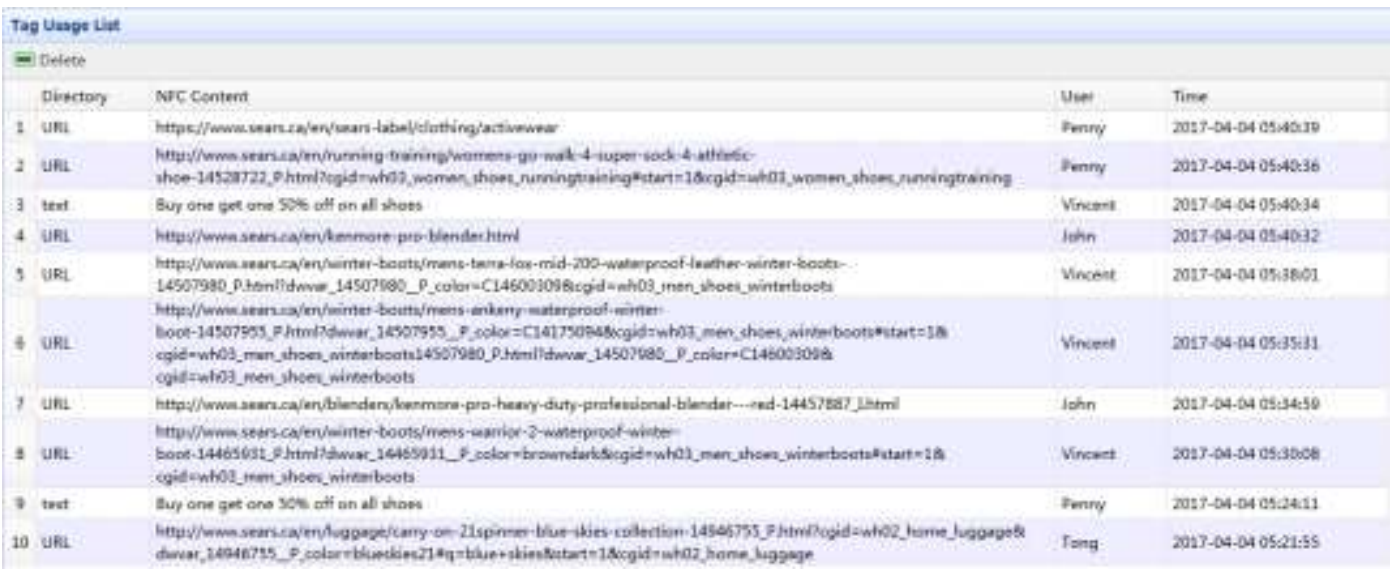

Figure 7. Tag Usage List

From the one page of the tag usage list (Figure 7) in the background server, we see there are four customers' tag read records, and we can try to summary personal shopping preference for each customer:

- Penny - activewear, running \& training shoes

- Vincent - winter boots

- John - blender

- Tong - luggage

According to the list, retailer can modify the existing marketing strategy such as customize deal for every customer, which will be much more flexible and effective than all-in-one activity. At the same time, it will make customers feel the retailer knowing about themselves and increase the sense of belonging. 


\section{Conclusion}

In this paper, the prototype of NFC Tag-based system is discussed with the NFC tag usage lifecycle, its complete architectural design and implementation. This system provides a mobile client based on Android platform to record NFC tag usage and send to database. To monitor and manage data, there is a background server for administrator. Besides, by utilizing the tag serial number, data collection and analysis becomes more diversified and flexible. Compare to the other NFC related system, we propose a new tag-based design concerning data collection and analysis data which is presented in the two case studies above. The first advantage of the prototype is that it creates a win-win model in the retail marketing. On the customer side, they have ability to choose some information which they truly care about based on personal purchase preference from tags. Because the mobile client in customer's device is only a tool to read information in tag, he or she does not have to be worried about spam messages or notifications. On the retailer side, NFC tag usage records have huge value to be excavate, such as customer's personal shopping behavior and compare between business competitors, which are all the most urgent need data in retail marketing. The other advantage is the characteristics of NFC tag. At first, it has widely implemented occasions. It can store various categories of content including Text, URL, Location, Contact and even Wi-Fi information, and can be read by most of NFC enabled devices. Secondly, NFC tag is incredibly cheap. It has very simple circuitry and few components. And then, as the security guarantee, the tag absorbs a small portion of the energy only when device approaches and starts sending modulated information. Last but not the least, it is very easy to manage such as edit, erase content or even lock or setup password by using the mobile client in our system. As is known to all, Near Field Communication has been implemented into many occasions in our life especially wireless payment combining with smartphone. However, NFC tag is always overlooked or limited used in marketing. The aim of the paper is excavating the its business value and developing an experimental prototype system targeting data collection and analysis.

\section{References}

[1] NFC Communication Post, “About Near Field Communication”, http://nearfieldcommunication.org/ about-nfc.html, (2017).

[2] R. Triggs, "All you need to know about NFC Tags", http://www.androidauthority.com/nfc-tags -explained-271872/, August 15, 2016

[3] D. Simões, "Loyalty Systems over Near Field Communication (NFC)"

[4] O. Busra, et al. "NFC loyal: A beneficial model to promote loyalty on smart cards of mobile devices." Internet Technology and Secured Transactions (ICITST), 2010 International Conference for. IEEE, (2010).

[5] M. Niehaus, "4 Problems with Customer Loyalty Programs", http://instoredoes.com/4-problems-withcustomer-loyalty-programs/, (2014). 